\title{
ARTICLE
}

Received 1 Dec 2013 | Accepted 17 Mar 2014 | Published 22 Apr $2014 \quad$ DOl: 10.1038/ncomms4668

\section{Co-option of a photoperiodic growth-phase transition system during land plant evolution}

\author{
Akane Kubota1, Shogo Kita1, Kimitsune Ishizaki ${ }^{1,2}$, Ryuichi Nishihama' ${ }^{1}$ Katsuyuki T. Yamato ${ }^{1,3}$ \& Takayuki Kohchi ${ }^{1}$
}

Photoperiodic control of the phase transition from vegetative to reproductive growth is critical for land plants. The GIGANTEA (GI) and FLAVIN-BINDING KELCH REPEAT F-BOX1 (FKF1) protein complex controls this process in angiosperms. However, little is known about how plants evolved this regulatory system. Here, we report that orthologues of GI and FKF1 are present in a basal plant, the liverwort Marchantia polymorpha, and describe the molecular interaction between their products. Knockout of either the GI or FKF1 orthologue completely abolishes the long-day-dependent growth-phase transition in $M$. polymorpha. Overexpression of either gene promotes growth-phase transition, even under short-day conditions. Introduction of the Gl orthologue partially rescues the late-flowering phenotype of the Arabidopsis thaliana gi mutant. Our findings suggest that plants had already acquired the GI-FKF1 system to regulate growth-phase transition when they colonized land, and that this system was co-opted from gametophyte to sporophyte generation during evolution.

\footnotetext{
${ }^{1}$ Graduate School of Biostudies, Kyoto University, Kyoto 606-8502, Japan. ${ }^{2}$ Graduate School of Science, Kobe University, 1-1 Rokkodai, Kobe 657-8501, Japan. ${ }^{3}$ Faculty of Biology-Oriented Science and Technology, Kinki University, Nishimitani, Kinokawa, Wakayama 649-6493, Japan. Correspondence and requests for materials should be addressed to T.K. (email: tkohchi@lif.kyoto-u.ac.jp).
} 
$\mathrm{P}$ erception of day length is the most reliable way for living organisms, including land plants, to predict seasonal changes. Throughout the life cycles of land plants, one of the most important events regulated by day length information is the transition from vegetative phase to reproductive phase. The molecular mechanism of the photoperiodic growth-phase transition has been extensively studied in angiosperms ${ }^{1-3}$. In the longday plant $A$. thaliana, GI and FKF1 play essential roles in the regulation of photoperiodic flowering ${ }^{4,5}$. GI is a large protein with domains of unknown function. It interacts with FKF1 to form a protein complex in the presence of blue light ${ }^{4}$. The GI-FKF1 complex is involved in the degradation of CYCLING DOF FACTOR1 (CDF1), CDF2, CDF3 and CDF5, which are transcriptional repressors of the flowering gene CONSTANS $(C O)^{6,7}$. Homologues of $G I$ and FKF1 have been reported in a wide variety of vascular plants from lycophytes to angiosperms 8 . The function of GI in the regulation of photoperiodic flowering has been shown to be common across several angiosperm species $^{9-13}$. However, neither GI nor FKF1 orthologues have yet been identified in fully sequenced non-vascular plants, including the moss Physcomitrella patens ${ }^{8}$. Therefore, it has been speculated that the GI and FKF1 genes emerged around the time of the divergence of vascular plants from non-vascular plants ${ }^{8}$. The photoperiodic growth-phase transition operates in diverse plant taxa, from bryophytes to angiosperms ${ }^{2,14,15}$; however, underlying molecular mechanisms in non-vascular plants remain unclear.

The liverwort $M$. polymorpha shows a clear photoperiodic growth-phase transition ${ }^{14,16}$. Liverworts represent a critical point in the early evolution of land plants, especially the water-toland transition ${ }^{17,18}$. In addition, molecular genetic tools including transformation techniques ${ }^{19-22}$ and strategies for targeted genome modification ${ }^{23,24}$ have been established for M. polymorpha. Here, we show that the GI-FKF1 complex is present in the gametophyte generation of $M$. polymorpha, and that it plays an important role in regulating photoperiodic growth-phase transition in this generation. Also, we demonstrate the equivalent functionality of GI between M. polymorpha and A. thaliana. These results give an insight into the evolution of the regulatory mechanism controlling photoperiodic growth-phase in land plants.

\section{Results}

Identification of GI and FKF1 orthologues in M. polymorpha. Under long-day conditions, $M$. polymorpha undergoes a transition from the vegetative to reproductive phase and initiates the formation of gametangiophores, which contain antheridia or archegonia, at the tips of gametophyte thalli $14,16,25,26$ (Supplementary Fig. 1). Exposure to far-red light (FR) is indispensable for this process ${ }^{19}$, suggesting the presence of light-quality- and day-length-dependent regulatory mechanisms in $M$. polymorpha. Interestingly, and in contrast to the aforementioned information, analysis of genome and transcriptome databases of $M$. polymorpha revealed single-copy orthologues for $G I$ and $F K F 1$, designated as $M p G I$ and $M p F K F$, respectively (Fig. 1). The intron insertion sites in the GI and FKF1 sequences are conserved between $M$. polymorpha and A. thaliana, with the exception of the first intron insertion sites of GI (Fig. 1a). MpGI is predicted to encode 1,187 amino-acid residues, with an estimated molecular mass of $127.5 \mathrm{kDa}$, thus showing similarity to GI homologues in vascular plants (Fig. 1b). MpFKF is predicted to encode 635 amino acids with an estimated molecular mass of $69.5 \mathrm{kDa}$. The sequence contains a LOV domain, F-box, and six tandem kelch motifs, similar to previously reported for FKF1 and its homologues, ZEITLUPE (ZTL) and LOV KELCH PROTEIN2 (LKP2) in A. thaliana ${ }^{27-29}$ (Fig. 1c). A cysteine residue that is involved in blue light perception via non-covalently binding to a chromophore, flavin mononucleotide (FMN), is conserved between MpFKF and A. thaliana FKF1 (ref. 5) (Fig. 1c). In a phylogenetic analysis, MpFKF formed a subgroup with an FKF homologue in Selaginella moellendorffi, which was independent from either the ZTL/LKP2 or FKF1 clade in angiosperms (Fig. 2).

In $A$. thaliana, the transcription of GI and FKF1 genes is regulated by the circadian clock ${ }^{27,30,31}$. Similarly, in mature thalli grown under 12-h-light/12-h-dark cycles, $M p G I$ and $M p F K F$ showed diurnal expression patterns, with phase peaks occurring around dusk (Fig. 3a,b). Furthermore, the rhythmic expression of $M p G I$ and $M p F K F$ persisted after the transition to constant light or dark conditions, suggesting that the expression of $M p G I$ and $M p F K F$ is regulated by the circadian clock (Fig. $3 \mathrm{c}-\mathrm{f}$ ).

Interaction between MpGI and MpFKF. We next examined complex formation between MpGI and MpFKF. Yeast two-hybrid analysis indicated an interaction between full-length proteins of MpGI and MpFKF (Fig. 4a). Truncation analysis revealed that the 392 amino-acid residues of the MpGI $\mathrm{N}$ terminus and the LOV domain of MpFKF were sufficient for the interaction (Fig. 4a). Coimmunoprecipitation of $3 \times$ FLAG-tagged MpGI with $4 \times$ myc-tagged MpFKF in transgenic plants suggests that the interaction between MpGI and MpFKF also occurs in vivo (Fig. 4b). Spatial expression analysis of $M p G I$ and $M p F K F$, using plants expressing the GUS reporter gene under the control of their promoter sequences, revealed overlapping GUS staining, which was observed continuously from the apical notch region to the midrib of mature thalli (Fig. $4 \mathrm{c}, \mathrm{d}$ ). These results suggest that the MpGI and MpFKF protein complex accumulates in the gametophyte of $M$. polymorpha.

Essential role of $\mathrm{MpGI}$ and $\mathrm{MpFKF}$ in growth-phase transition. To investigate the physiological function of the MpGI-MpFKF complex in $M$. polymorpha, we generated knockout mutants of both $M p G I$ and $M p F K F$ using a homologous recombinationmediated gene targeting strategy ${ }^{23}$. Disruption of these genes was performed by replacing the second exon of $M p G I$ and a 264-bp region of $M P F K F$, including the conserved FMN-binding domain, with a hygromycin-resistant marker (Supplementary Fig. 2a,b). Genomic PCR analysis confirmed the homologous recombination events on the MpGI and MpFKF loci, in the $g i^{k o}$ and $f k f^{k o}$ lines, respectively (Supplementary Fig. 2c,d). RT-PCR analysis revealed that $M p G I$ and $M p F K F$ gene expression was undetectable in these lines (Supplementary Fig. 2e,f). Ten-day-old wild-type and knockout thalli grown from gemmae under constant light conditions were subjected to long-day conditions supplemented with FR. Under these conditions, individual wild-type plants formed the first visible gametangiophore within 20 days, whereas neither $g i^{k o}$ nor $f k f^{k o}$ plants formed any gametangiophores after 60 days, indicative of a failure of the growth-phase transition in the knockout lines (Fig. 5a,b). These defects were rescued by introducing the genomic fragment of $M p G I$ or $M p F K F$ (Fig. 5a,b, and Supplementary Fig. 2e,f). This indicates that the $M p G I$ and $M P F K F$ genes are required for growth-phase transition under long-day conditions. Given that MpGI could interact with $\mathrm{MpFKF}$ in vivo, and that a single disruption of $M p G I$ or $M p F K F$ caused equally severe defects in the growth-phase transition without mutually affecting their transcription (Supplementary Fig. 2g), these observations suggest that formation of the MpGI and MpFKF complex is critical for growth-phase transition in M. polymorpha.

Effect of overexpression of MpGI or MpFKF on photoperiodism. In $A$. thaliana, simultaneous overexpression of 
a

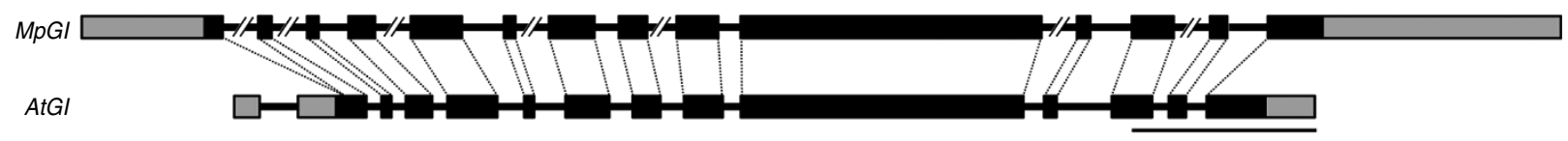

MPFKF

AtFKF1

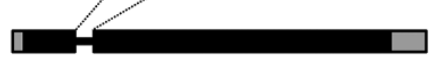

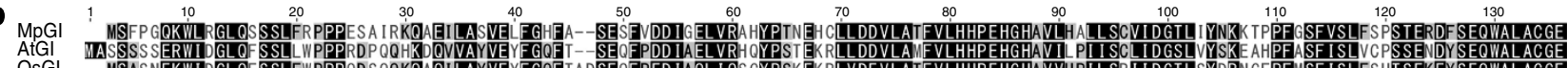

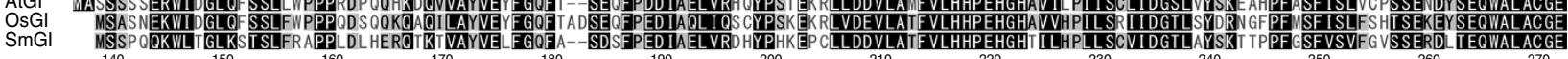

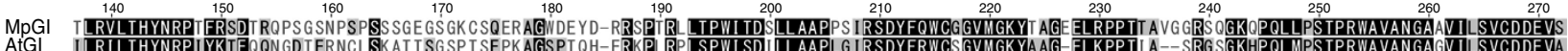

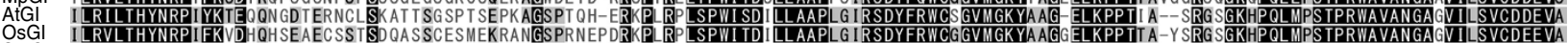

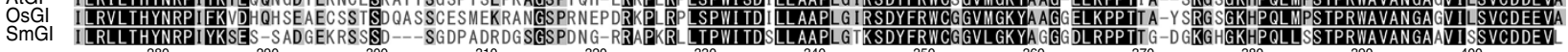

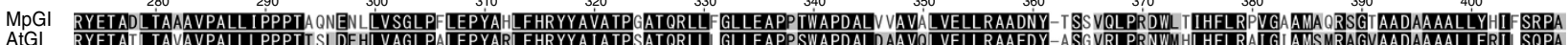

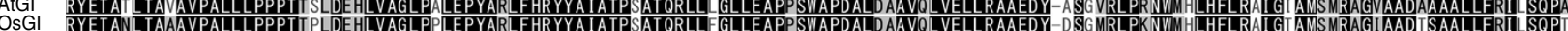

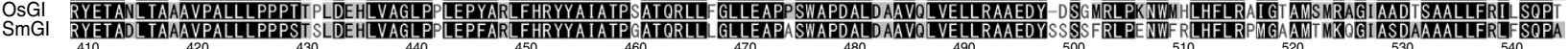

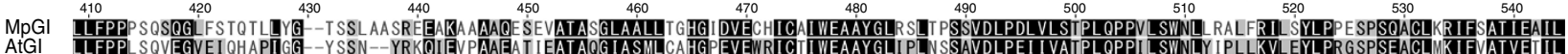

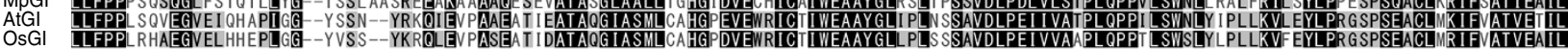

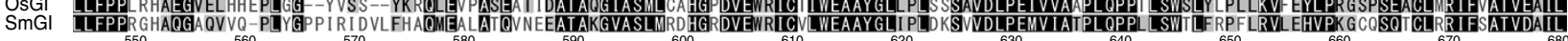

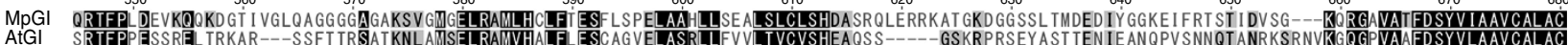

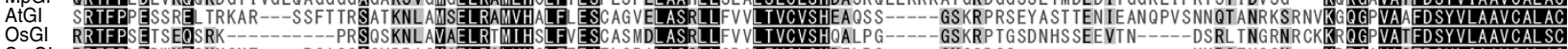

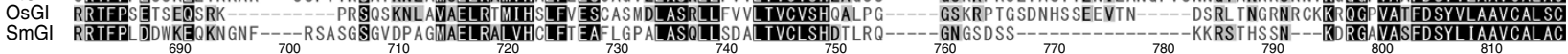

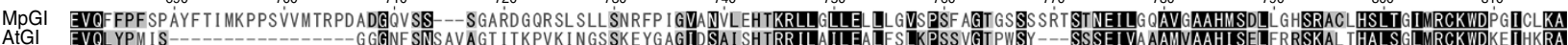
AtGI EVQLYPMIS-- IS

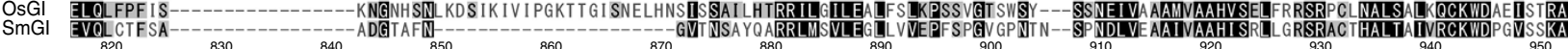

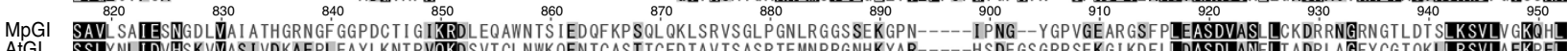

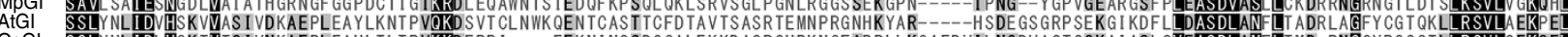

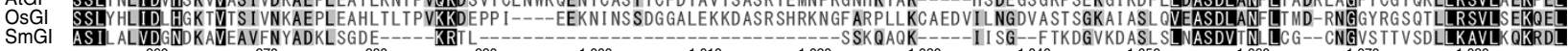

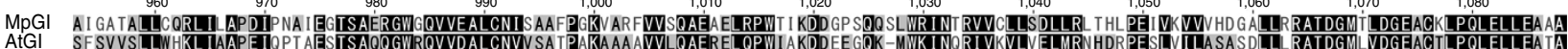

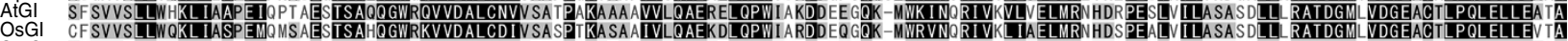

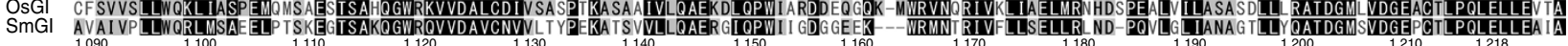

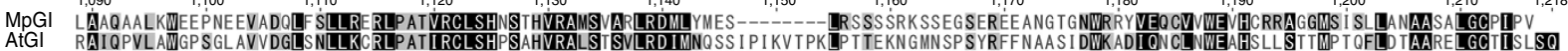

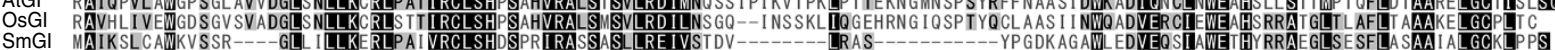

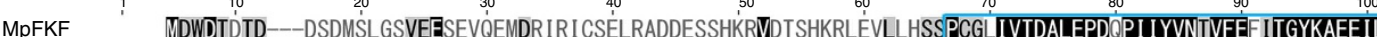
AtFKF1 WAREHAIGEATGKRKKRGRVEEAEEYCNDGIE---EQVEDEKLPLEVGM-----FYYPMTPPSFIVSDALEPDFPLIYVNRVFEVFTGYRADEVI AtZTL WEWDSGSDLSADDASSLADDEEGGLFPGG-_-_-_PIPYPVGN-_-_-_LLTAPCGFVVTDAVEPDOPIIYVNTVFEMVTGYRAEEVL AtLKP2 WONOMEWDSDSDLSGGD----EVAEDGWFGGDN--_-_-_AIPFPVGS---_-LPGTAPCGFVVSDALEPDNPIIYVNTVFEIVITGYRAEEVI

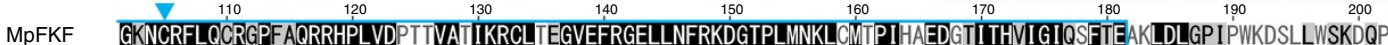
AtFKF1 GRNCRFLOYRDPRAQRRHPLVDPVVVSEIRRCLEEGIEFQGELLNFRKDGTPLVNRLRLAPIRDDDGTITHVVIGIQVESETTTIDLDRVSY-AtZTL GGNCRFLOCRGPFAKRRHPLVDSMVSEIRKCIDEGIEFQGELLNFRKDGSPLMNRLRLTPIYGDDDTITHIIGIQFFIETDIDLGPV--_-_GSSTKE AtLKP2 GRNCRFLQCRGPFTKRRHPMVDSTIVAKMROCLENGIEFQGELLNFRKDGSPLMNKLRLVPIREEDE-ITHFIGVLLFTDAKIDLGPSPD----LSAKEIP

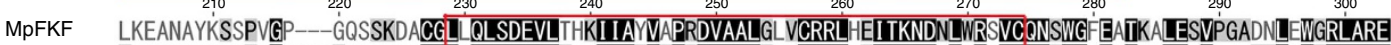
AtFKF1 QLDQTSECLFPSG̈SPRFKEHHEDFCGIL QRLSDEVLAHNILSRLTPRDVASIGSACRRLRQLTKNESVRKM VCONAWGKEI ITGTLEI II-TKKLRWGRLARE AtZTL KSIDGIYSALAAGE---RNVSRGMCGLFOLSDEVVSUKILSRLTPRDVASVSSVGRRLYYLTKNEDLWRRVCONAWGSETTRVLETVPGAKRLGWGRLARE AtLKP2 RISRSFTSALPIGE---RNVSRGLCGIFELSDEVIAIKILSQLTPGDIASVGCVGRRLNELTKNDDVWRMVCONTWGTEATRVLESVPGAKRIGWVRLARE

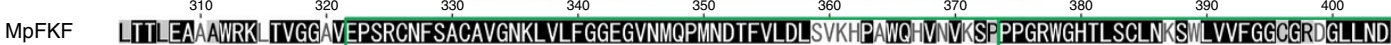
AtFKF1 LTTLEAVCWRKFTVGGI VOPSRCNFSACAVGNRLVLFGGEGWMOPLDDTFVLNLDAECPEWORVRVTSS PPGRWGHTLSCLNGSWLVVFGGCGROGLLND AtZTL LTTLEAAAWR L SVGGSVEPSRCNFSACAVGNRVVLFGEGVNMOPMNDTFVLDLNSDYPEWQHVKVSSPPPGRWGHTLTCVNGSNLVVFGGCGQOGLLND AtLKP2 FTTHEATA AWRKFSVGGTVEPSRCNFSACAVGNRIVIFGGEGVNMOPMNDTFVLDLGSSSPEEWKSVILVSSPPPGRWGHTLSCVNGSRLVVFGG YGSHGGLLND

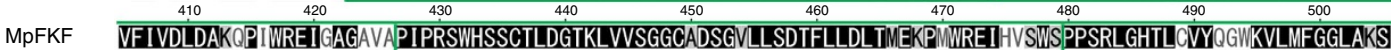
AtFKF1 VFVLDLDAKHPTWKEV-AGGTPPLPRSWHSSCTIEGSKLVVSGGCTDAGVLLSDTFLLDLTTDKPTTKEIPTSWAPPSRLGHSLSVFGRTKILMFGGLANS AtZTL VFVLNLDAKPPTWREI-SGLAPPLPRSWHSSCTLDGTKLIVSGGCADSGVLLSDTFLLDLSIEKPVWREIPAAWTPPSRLGHTLSVYGGRKILMFGGLAKS AtLKP2 VFLLDLDADPPSWREV-SGLAPPIPRSWHSSCTLDGTKLIVSGGCADSGALLSDTFLLDLSMDIPAWREIPVPWTTPSRLGHTLTVYGDRKILMFGGLAKN

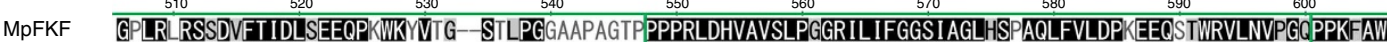
AtFKF1 GHULLRSGEAYTIDLEDEEPRWRELEC--SAFPGVV----VPPPRLDHVAVSWPCGRVIIFGGSIAGLHSP SOLFLIDPAEEKPSWRILNVPGK PPKLAW AtZTL GPLKFRSSDVFTMDLSEEEEPCWRCVTG--SGMPGAGNPGGVAPPPRLDHVAVMLPGGRILIFGGSVAGLHSASOLYLLDPTEDKPTWRILNIPGRPPRFAM AtLKP2 GTLRFRSNDVYTMDLSEDEPSWRPVI GYGSSLPGGMA---APPPRLDHVAISLPGGRILIFGGSVAGLDSASOLYLLDPNEEKPAWRILNVOGGPRFAW MpFKF $\quad \frac{610}{\text { GHSTCVVGGTRAVVLGGHTGEEWILN--ELHELSITHKSLSSTZ }}$ AtFKF1 GHSTCVVGGTRVLVLGGHTGEEWILN--ELHELCLASRQDSDL

AtZTL GHATCVVGGTRAIVLGGOTGEEWWLRYWSFRGERLSGGTLVLLI IFFSFFFFLPH

AtLKP2 GHTTCVVGGTRLVVLGGQTGEEEWILN--EAHELLLATSTTAST

Figure 1 | Primary structures of MpGI and MpFKF. (a) Gene structure of $G I$ and FKF1 homologues in M. polymorpha and A. thaliana. Black boxes, grey boxes and black lines indicate exons, untranslated regions and introns, respectively. Scale bar, $1 \mathrm{~kb}$. Introns present in $M$. polymorpha genes are connected to the corresponding regions in $A$. thaliana genes by dashed lines. (b,c) Alignment of amino-acid sequences from GI (b) and FKF1 (c) homologues in M. polymorpha, A. thaliana, O. sativa and S. moellendorffii. Identical and similar amino-acid residues are highlighted and shaded, respectively. The conserved cysteine residue involved in FMN binding is indicated by a blue triangle. Blue, red and green open boxes indicate LOV domain, F-box and kelch motifs, respectively. 


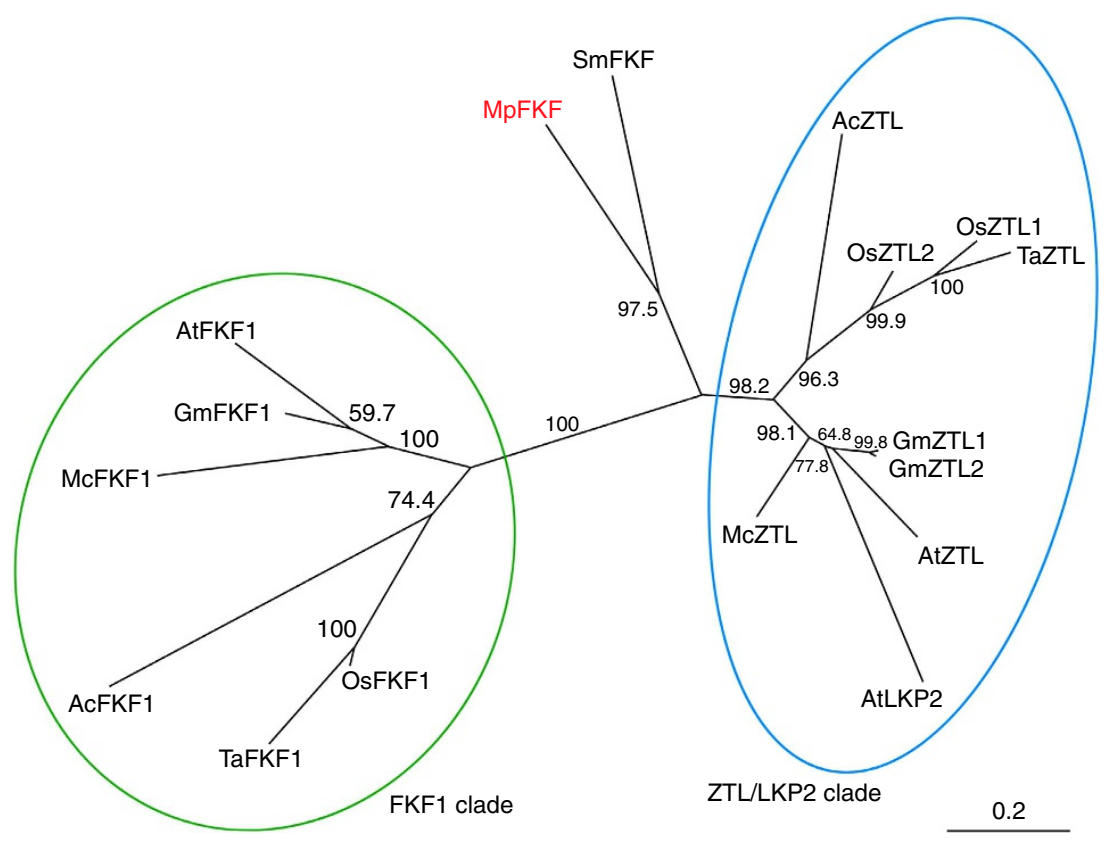

Figure 2 | Phylogenetic analysis of MpFKF. An unrooted maximum-likelihood phylogenetic tree was estimated using amino-acid sequences of FKF/ZTL/ LKP2 homologues. Protein sequences used for the analysis were from A. thaliana (AtFKF1, AtZTL, and AtLKP2), O. sativa (OsFKF1, OsZTL1 and OsZTL2), Glycine max (GmFKF1, GmZTL1 and GmZTL2), Triticum aestivum (TaFKF1 and TaZTL), Allium cepa (AcFKF1 and AcZTL), Mesembryanthemum crystallinum (McFKF1 and McZTL) and S. moellendorffii (SmFKF). The clade that consists of FKF homologues is marked with green, and the clade consisting of LKP2/ZTL homologues with blue. Scale bar, evolutionary distance in substitutions per residue. Numbers, bootstrap probabilities.

GI and FKF1 increases the amount of GI-FKF1 complex and eliminates photoperiodicity to promote flowering under short-day conditions ${ }^{4}$. To examine whether overexpression of MpGI and MpFKF alters photoperiodic growth-phase transition in $M$. polymorpha, we generated overexpression lines of MpGI-FLAG or MpFKF-myc under the control of an M. polymorpha ELONGATION FACTOR1- $\alpha$ (MpEF) promoter ${ }^{32}$. In wild-type plants, gametangiophore formation is delayed under short-day conditions (Fig. 5d and Supplementary Fig. 1), whereas the number of days required for gametangiophore formation in the overexpression lines of either gene was significantly shortened and was comparable to those of wildtype plants grown under long-day conditions (Fig. 5c,d). In addition, the growth-phase transition of these overexpression lines under long-day conditions was slightly promoted (Fig. 5d). Simultaneous overexpression of MpGI-FLAG and MpFKF-myc did not enhance the phenotype caused by single-gene over expression, suggesting that MpGI and MpFKF may act genetically in the same pathway. These results suggest that overexpression of $M p G I$ or $M p F K F$ could increase the amount of MpGIMpFKF complex and promote the growth-phase transition regardless of photoperiod. Overexpression of only MpGI-FLAG in the $f k f^{k o}$ background or only MpFKF-myc in the $g i^{k o}$ background did not promote growth-phase transition under short-day conditions within 3 weeks. In contrast, all overexpression lines in the wild-type background formed gametangiophores within 3 weeks (Fig. 5d). These findings supported our hypothesis that MpGI and MpFKF co-require each other and function as a complex. It is noteworthy that under white light conditions without FR supplementation, no gametangiophore formation was observed by day 60 in any of the overexpression lines. Therefore, the MpGI- and MpFKFmediated photoperiodic pathway is likely to act independently of the light-quality-dependent pathway to regulate growth-phase transition in M. polymorpha.
Complementation of Arabidopsis gi with MpGI. Our findings showed that the regulatory system of photoperiodic growth-phase transition mediated by GI-FKF1 is present in the gametophyte of liverwort. To examine whether $M p G I$ is functionally equivalent to angiosperm $G I$ and able to function in the regulation of flowering, we introduced $M p G I$ into an $A$. thaliana gi mutant ${ }^{30,31}$. Expression of MpGI under the control of the AtGI promoter partially complemented the late-flowering phenotype of the A. thaliana gi mutant (Fig. 6), suggesting that the MpGI gene has the potential to act as a regulator of flowering.

\section{Discussion}

In this study, we demonstrated that both $M$. polymorpha and A. thaliana use the GI-FKF1 system to regulate photoperiodic growth-phase transition. This suggests that the molecular mechanisms regulating gametangiophore formation in the liverwort are equivalent to those involved in flowering in angiosperms. In $A$. thaliana, the GI-FKF1 complex directly degrades CDF1 and regulates flowering time via transcriptional regulation of the flowering genes CO and FLOWERING LOCUS T $(F T)^{1}$. Although these genes that regulate flowering are widely conserved in angiosperms ${ }^{2}$, a previous report on $C O$ homologues in $P$. patens showed that they are more closely related to CO-like genes, AtCOL3/AtCOL4/AtCOL5, which form a distinct group from $\mathrm{CO}^{33}$. Also, the presence of FT is controversial. In the phosphatidylethanolamine-binding protein (PEBP) gene family, the family to which FT and TERMINAL FLOWER1 (TFL1) belong ${ }^{34,35}$, the genes in the FT/TFL1 subfamily were suggested to have arisen in seed plants, since they are absent from the genomes of $P$. patens and $S$. moellendorffi ${ }^{36}$. Identification of the growthphase transition regulators that are downstream of the MpGI-MpFKF complex will greatly advance our understanding of how photoperiodic growth-phase regulation mechanisms evolved in land plants. 

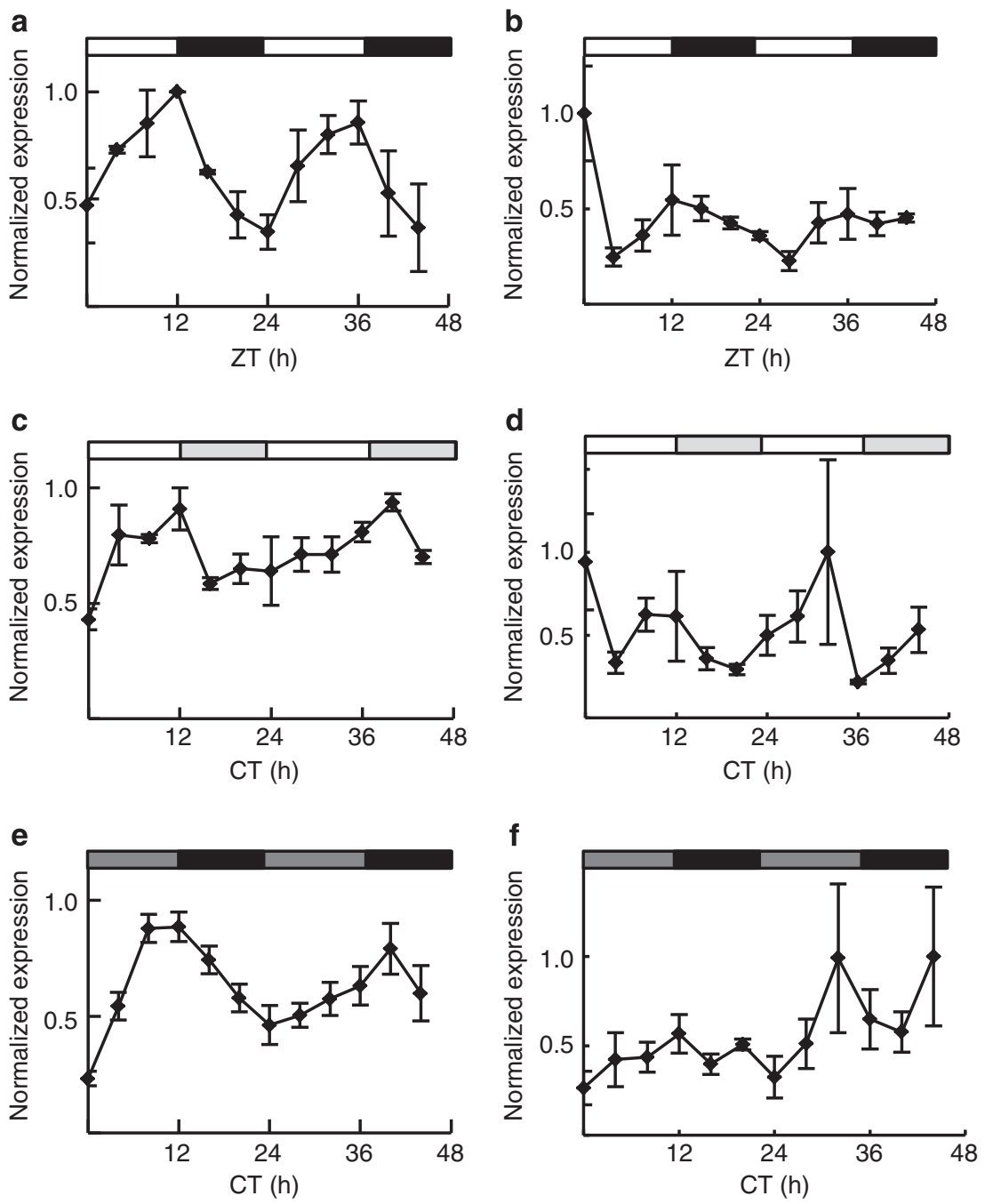

Figure 3 | Circadian expression of $\mathbf{M p G I}$ and $\mathbf{M p F K F}$ in $\boldsymbol{M}$. polymorpha. (a-f) Transcript accumulation profiles of $M p G I(\mathbf{a}, \mathbf{c}, \mathbf{e})$ and $M p F K F(\mathbf{b}, \mathbf{d}, \mathbf{f})$ monitored under a 12-h-light/12-h-dark cycle $(\mathbf{a}, \mathbf{b})$, constant light $(\mathbf{c}, \mathbf{d})$ and constant dark (e,f) conditions. Bars above the traces represent light conditions; open, filled, lightly shaded and darkly shaded bars represent day, night, subjective night and subjective day, respectively. ZT, Zeitgeber time; $\mathrm{CT}$, circadian time (time after the transfer from light-dark cycles to constant conditions). Error bars, s.e.m.; $n=3$.

The presence of the GI-FKF1 complex in the liverwort lineage strongly suggests that this system had already been acquired in the common ancestor of land plants. Sequencing of the genomes of species in the Charophyceae, a class with a sister relationship to land plants ${ }^{17}$, as well as comparative genomic approaches will be required to further understand the evolutionary novelty of the GI-FKF1 system during the course of green plant evolution.

Interestingly, the GI-FKF1 system functions in the gametophyte generation in M. polymorpha, whereas it functions in the sporophyte generation in A. thaliana. These facts imply that, during the course of land plant evolution, the GI-FKF1 system that evolved to regulate reproductive phase transition in the gametophyte generation in basal land plants was co-opted to regulate that in the sporophyte generation in vascular plants.

\section{Methods}

Plant material and growth conditions. Male and female accessions of M. polymorpha, Takaragaike-1 (Tak-1) and Takaragaike-2 (Tak-2), respectively ${ }^{20}$ were used as the wild type. Plants were cultured axenically on half-strength Gamborg's B5 medium ${ }^{37}$ containing $1 \%$ sucrose and $1.3 \%$ agar, under a $50-60 \mu \mathrm{mol} \mathrm{m}^{-2} \mathrm{~s}^{-1}$ continuous white cold cathode fluorescent lamp (CCFL; OPT-40C-N-L, Optrom) at $22{ }^{\circ} \mathrm{C}$, unless otherwise defined. For analysis of growth-phase transition, gametangiophores were induced in growth chambers (LH-80CCFL-6CT, NKSystem) under long-day (16-h-light/8-h-dark) or short-day (8-h-light/16-hdark) conditions using $40-60 \mu \mathrm{mol} \mathrm{m}{ }^{-2} \mathrm{~s}^{-1}$ CCFL supplemented with $20-$ $30 \mu \mathrm{mol} \mathrm{m}^{-2} \mathrm{~s}^{-1} \mathrm{FR}$ light-emitting diodes (VBL-TFL600-IR730*, Valore; peak emission at $733 \mathrm{~nm}$, with a full width at half maximum of $27 \mathrm{~nm}$ ) at $22^{\circ} \mathrm{C}$

Sequence retrieval and phylogenetic analysis. The search for $M$. polymorpha GI and FKF1 gene homologues was performed using the transcriptome and genome databases from the M. polymorpha on-going Joint Genome Institute genomesequencing project (http://www.jgi.doe.gov/). Protein alignments were constructed using MUSCLE alignment implemented in the Geneious 6.1.6 software package (Biomatters; http://www.geneious.com) at the default parameters, and an unrooted maximum-likelihood phylogenetic tree constructed using PhyML program ver. 2.2.0 (ref. 38) implemented in the Geneious software, using the LG model and four categories of rate substitution. Tree topology, branch length and substitution rates were optimized, and the tree topology was searched using the nearest-neighbour interchange method. Bootstrap values were computed from 1,000 trials. The sequences used for the protein alignment of GI homologues were AtGI (ABP96497) from A. thaliana, OsGI (Os01g0182600) from Oryza sativa and SmGI (XP_002961231) from S. moellendorffi. The sequences used in the phylogenetic tree of FKF1/ZTL/LKP2 were as follows: AtFKF1 (AAF32298), AtZTL (NP_001154783), AtLKP2 (NP_849983) from A. thaliana; OsFKF1 (Os11g0547000), OsZTL1 (Os02g0150800), OsZTL2 (Os06g0694000) from O. sativa; GmFKF1 (NP_001235886), GmZTL1 (NP_001235856), GmZTL2 (NP_001235871) from Glycine max; TaFKF1 (ABL11478), TaZTL (ABR14627) 


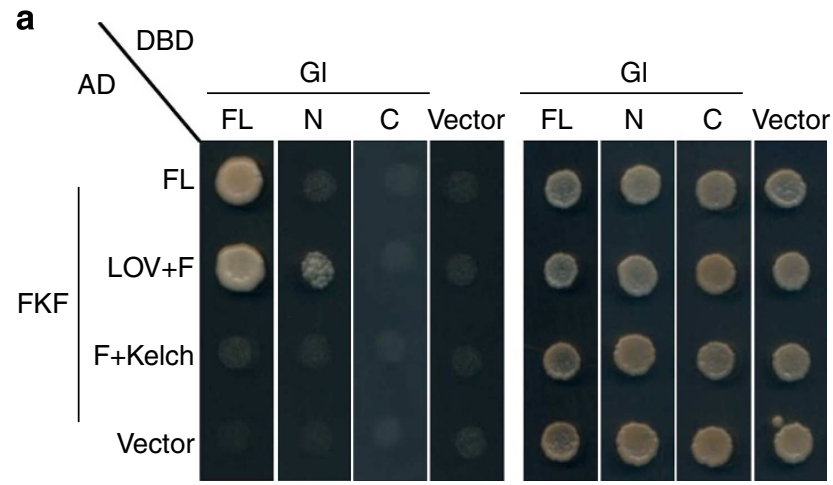

b

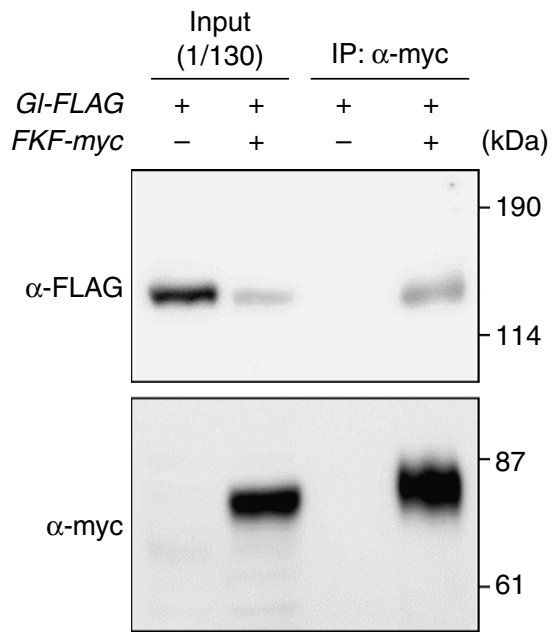

C

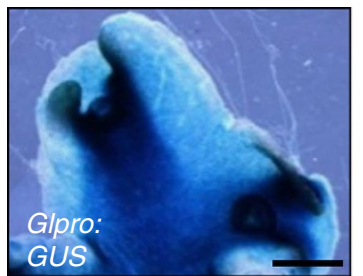

d

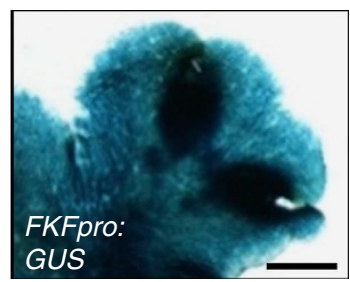

Figure 4 | Interactions and spatial expression of MpGI and MpFKF.

(a) Yeast two-hybrid analysis. Full-length (FL) or truncated MpGI and MpFKF fused to the DNA-binding domain (DBD) or the activation domain $(A D)$ of Gal4 were tested under selective ( $-\mathrm{WLH}$, left) and non-selective ( $-\mathrm{WL}$, right) conditions. For $\mathrm{MpGl}, \mathrm{N}$ and $\mathrm{C}$ indicate the amino-acid residues 1-392 and 383-1,187, respectively. For MpFKF, F and Kelch indicate F-box and kelch-repeat domains, respectively. (b) Co-immunoprecipitation of GI-FLAG and FKF-myc from the extracts of plants overexpressing MpGI-FLAG only or both MpGI-FLAG and MpFKF-myc. Anti-FLAG $(\alpha-F L A G)$ and anti-c-Myc $(\alpha-m y c)$ antibodies were used to detect MpGI-FLAG and MpFKF-myc, respectively, by western blotting. (c,d) GUS staining images from 10-day-old plants harbouring MpGlpro:GUS (c) or MpFKFpro:GUS (d). Scale bar, $1 \mathrm{~mm}$.

from Triticum aestivum; AcFKF1 (ACT22762), AcZTL (ACT22763) from Allium сера; McFKF1 (AAQ73528), McZTL (AAQ73527) from Mesembryanthemum crystallinum; and SmFKF (XP_002974336) from S. moellendorffii.

Plasmid construction. To generate the MpGI-targeting vector, 3.5-kb homologous arms were amplified from Tak-1 genomic DNA by PCR using KOD FX Neo (Toyobo) with the primer pairs: forward 5'-CTAAGGTAGCGATTAATGCAGGA ATAGTAGAGATCATGAAGG- $3^{\prime}$, reverse $5^{\prime}$-CCGGGCAAGCTTTTAATCGTG AAACGATCCCAAATCT- $3^{\prime}$ (for the $5^{\prime}$ side); and forward $5^{\prime}$-AACACTAGTGGC GCGAGCCAAAGAACCGTATCGAA-3', reverse $5^{\prime}$-TTATCCCTAGGCGCGTG
GCATTCCATTCTATGCTG-3' (for the $3^{\prime}$ side). The PCR-amplified fragments of the $5^{\prime}$ end and $3^{\prime}$ end were cloned into the PacI site and AscI sites of pIHY-TMp1 (ref. 23), respectively, using an In-Fusion HD cloning kit (Clontech). The MpFKFtargeting vector was designed in the reverse direction to that of MpGI. 6-kb homologous arms that were amplified using the primer pairs: forward $5^{\prime}$-TTATCC CTAGGCGCGCACACTCCGTGCAATCTCAA- ${ }^{\prime}$, reverse $5^{\prime}$-TAAACTAGTGG CGCGCCACAACGATCCTACCACATC- $3^{\prime}$ (for the $5^{\prime}$ side), and forward $5^{\prime}$-GCC CGGGCAAGCTTAACCATGGAAGGACTCACTGC- ${ }^{\prime}$, reverse $5^{\prime}$-CTAAGGTA GCGATTAAGTTCGGACAGCTTCCTTCA- $3^{\prime}$ (for the $3^{\prime}$ side), and cloned into the AscI site and PacI site of pJHY-TMpl (ref. 23), respectively. The resultant targeting vectors were introduced into $\mathrm{F}_{1}$ sporelings of $M$. polymorpha derived from crosses between Tak-1 and Tak-2, as described previously ${ }^{20}$. Screening for gene-targeted lines was performed by genomic PCR as described previously ${ }^{23}$. Gene-specific primers used in the screening are illustrated in Supplementary Fig. 2a,b and Supplementary Table 1. For the complementation test of $g i^{k o}$ and $f k f^{k o}$, genomic fragment including entire regions of $M p G I$ and $M p F K F$ was amplified from Tak-1 genomic DNA by PCR using KOD Plus Neo (Toyobo) with the primer pairs: forward $5^{\prime}$-CACCGCTATTCTTCACGCCGAAA- $3^{\prime}$, reverse $5^{\prime}$-TCCCCCTTTATTGTTGAACT-3' (for $M p G I$ ), and forward $5^{\prime}$-CACCTGACC GTTTAATTCATTGTGGA- $3^{\prime}$, reverse $5^{\prime}$-GATGGTTTCCCGTACAGGTG- $3^{\prime}$ (for $M p F K F$ ), and cloned into pENTR/D-TOPO (Life Technologies). These were used to generate binary plasmids harbouring MpGIpro:gMpGI or MpFKFpro:gMpFKF. The resultant plasmids were introduced into corresponding knockout mutants, $g i{ }^{k o}$ or $f k f^{k o}$, as described previously ${ }^{21}$. To construct MpGIpro:GUS and MpFKFpro:GUS, a 4,573-bp fragment of $M p G I$, and a 5,082-bp fragment of $M p F K F$, as measured from their predicted start codons, were amplified from Tak-1 genomic DNA by PCR using KOD Plus Neo with the primer pairs: forward 5'-CACCAGATTTTAGGTTGACTCG- ${ }^{\prime}$, reverse $5^{\prime}$-GGGAAATGACATAGGG CCAA- $3^{\prime}$ (for $M p G I$ ), and forward $5^{\prime}$-CACCTGACCGTTTAATTCATTGT GGA-3', reverse $5^{\prime}$-ACCCAAGGACATATCCGAGT-3' (for $M p F K F$ ), respectively. Each fragment was cloned into pENTR/D-TOPO and used to generate binary plasmids harbouring MpGIpro:GUS and MpFKFpro:GUS, which were introduced into $F_{1}$ sporelings generated from Tak-1 and Tak-2 (ref. 20). To generate overexpression lines of $M p G I$ and $M p F K F$, full-length cDNA of $M p G I$ and $M p F K F$ was amplified using the primer pairs: forward $5^{\prime}$-CACCATGTCATTTCCCGGG CAGAA- $3^{\prime}$, reverse $5^{\prime}$-GACAGGAATCGGGCAGC-3' (for $M p G I$ ), and forward 5'-CACCATGGATTGGGATACAGATACCG-3', reverse 5'-GGTGCTAGACAGA CTCTTATGTGTTA- $3^{\prime}$ (for $M p F K F$ ). These were fused with 3xFLAG or 4 xmyc sequences to generate binary plasmids harbouring MpEFpro:MpGI-3xFLAG and MpEFpro:MpFKF-4xmyc. These overexpression constructs were introduced into cut thalli of Tak-1 as described previously ${ }^{21}$. Transformants, after two cycles of transplantation of gemmae, were used for the remainder of the experiments.

Total RNA isolation and cDNA synthesis. Tak-1 gemmae were grown under constant light for 9 days, followed by a 12-h-light/12-h-dark cycle for 6 days. From day 16, plants were harvested and immediately frozen in liquid nitrogen at 4-h intervals over 2 days under specific light conditions described in the text for each experiment. The frozen samples were ground into a frozen powder by shaking for $5 \mathrm{~min}$ with $6-\mathrm{mm}$ stainless beads in collection tubes using a ShakeMaster (BMS 12-NA, BioMedicalScience). Total RNA was extracted from the powder using TRIzol (Life Technologies) and treated with RQ1 RNase-Free DNase (Promega). For each sample, $4 \mu \mathrm{g}$ RNA was reverse transcribed to cDNA using random hexamer primers and ReverTra Ace (Toyobo), following the manufacturer's instructions.

Quantitative RT-PCR analysis. cDNA samples were diluted 1:4 and amplified with the CFX96 Real-Time PCR Detection System (Bio-Rad) using SYBR Green I Nucleic Acid Gel Stain (Lonza) to monitor dsDNA synthesis. Primer sequences and their optimal melting temperatures are listed in Supplementary Table 1. The three-step PCR cycling program was as follows: $95^{\circ} \mathrm{C}$ for $2 \mathrm{~min}$, followed by 40 cycles at $95^{\circ} \mathrm{C}$ for $10 \mathrm{~s}$, melting temperature for $15 \mathrm{~s}$ and $72^{\circ} \mathrm{C}$ extension for $15 \mathrm{~s}$. Melt-curve analysis was performed to ensure specificity of the products. Each cDNA sample was run at least in duplicate. PCR efficiencies were calculated using the software CFX Manager (Bio-Rad) in accordance with the manufacturer's instructions. Raw expression data were normalized using $M p E F$ expression data, and peak levels were set to 1 . The relative expression values of each gene were calculated to show the average \pm s.e. of mean of three biologically independent trials. Expression of the $M p E F$ reference gene showed constant patterns, without circadian rhythmicity, over all sample time points (data not shown). All primers used for the analysis are listed in Supplementary Table 1.

Co-immunoprecipitation experiments. Plants were grown under constant light conditions for 10 days, transferred to FR-supplemented long-day conditions for a further 7 days and harvested at ZT12 of day 18 for use in the experiments. Approximately $500 \mathrm{mg}$ of plants were harvested, frozen in liquid nitrogen and ground into a frozen powder. The protein was extracted in $1 \mathrm{ml}$ of ice-cold IP buffer ( $50 \mathrm{mM}$ Tris-HCl pH 7.4, $5 \mathrm{mM}$ EDTA, $50 \mathrm{mM} \mathrm{NaCl}, 1 \mathrm{mM}$ dithiothreitol, $0.1 \%(\mathrm{v} / \mathrm{v})$ Triton X-100, $10 \%(\mathrm{v} / \mathrm{v})$ glycerol, $50 \mu \mathrm{M}$ MG-132 and cOmplete protease inhibitor (Roche)). After centrifugation at $3,500 \mathrm{~g}$ for $2 \mathrm{~min}$ and $16,400 \mathrm{~g}$ for 
a

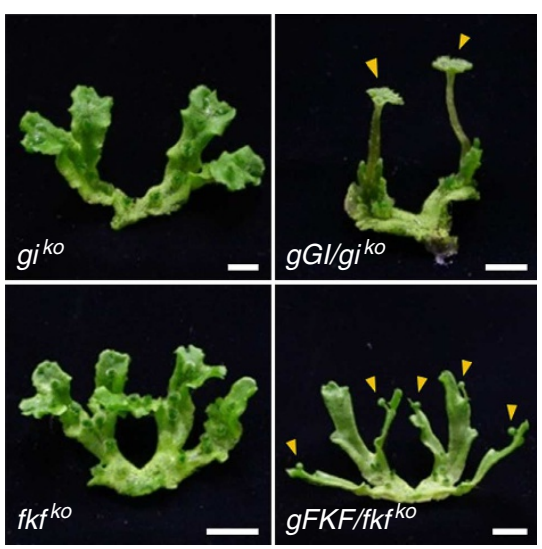

C

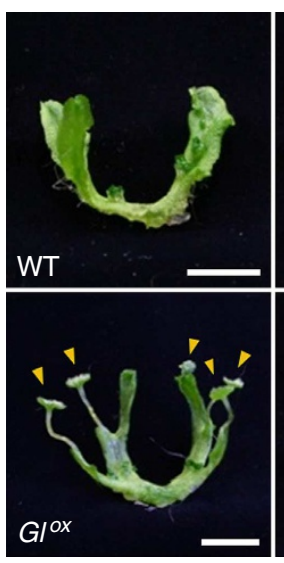

b

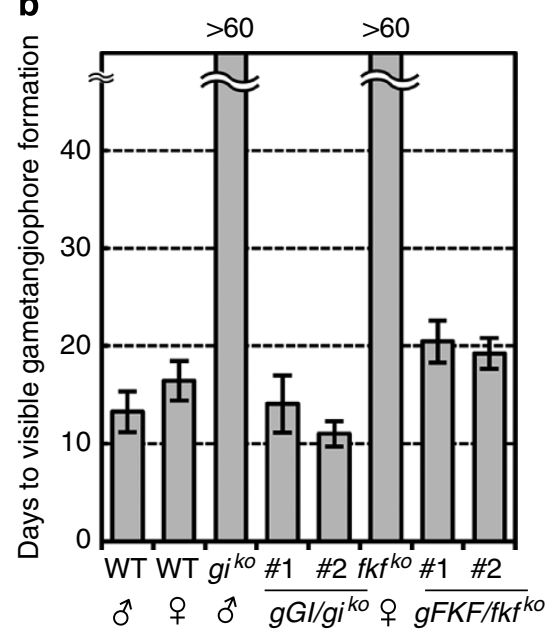

d

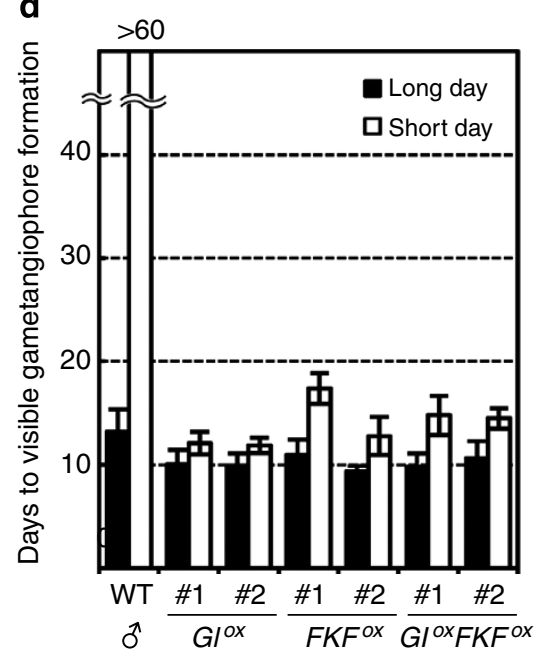

Figure 5 | Effects of disruption and overexpression of $\mathbf{M p G I}$ and $\mathbf{M p F K F}$ on the growth-phase transition of $\boldsymbol{M}$. polymorpha. (a,b) Timing of gametangiophore formation in wild-type plants, knockout mutants of $M p G I\left(g i k o\right.$, male) and $M p F K F\left(f k f^{k o}\right.$, female) and their complementation lines ( $g G l / g i^{k o}$ and $g F K F / f k k^{k o}$; two lines for each). Plants were grown from gemmae under long-day conditions. (c,d) Timing of gametangiophore formation in wild-type plants and lines overexpressing $M p G I\left(G I^{0 x}\right), \operatorname{MpFKF}\left(F K F^{\circ x}\right)$ or both $\left(G 1^{0 x} F K F^{O x}\right)$. Plants were grown from gemmae under long- and short-day conditions. Photographs of 35-day-old plants $(\mathbf{a}, \mathbf{c})$ and days to the first visible gametangiophore formation $(\mathbf{b}, \mathbf{d})$ are shown. Arrowheads indicate gametangiophores. WT, wild type. Scale bar, $10 \mathrm{~mm}$. Error bars, s.d.; $n>15$.

$10 \mathrm{~min}$ to remove cell debris, the supernatant containing approximately $5 \mathrm{mg}$ of total protein was mixed with $50 \mu \mathrm{l}$ of magnetic beads conjugated to an anti-myc antibody (Miltenyi Biotech) and then incubated on ice for $30 \mathrm{~min}$. The mixtures were applied to $\mu$ Columns (Miltenyi Biotech) in a magnetic field. After 10 washes with $200 \mu \mathrm{l}$ of IP buffer and one rinse with $150 \mu \mathrm{l}$ of Wash Buffer 2 (Miltenyi Biotech), proteins were eluted using $90 \mu \mathrm{l}$ of $2 \times$ sample buffer $(60 \mathrm{mM}$ Tris-HCl $\mathrm{pH} 6.8,5 \%$ sodium dodecyl sulphate (SDS), $20 \%(\mathrm{v} / \mathrm{v})$ glycerol, and $10 \%(\mathrm{v} / \mathrm{v}) 2-$ mercaptoethanol) to obtain the bound fraction. The immunoprecipitates were then subjected to immunoblot analysis.

Immunoblot analysis. Proteins were size fractionated using 8\% SDS polyacrylamide gel electrophoresis, followed by protein gel blot analysis using a 1:10,000 dilution of anti-FLAG M2 IgG antibody (F1804, Sigma-Aldrich) and a 1:500 dilution of anti-c-Myc IgG antibody (014-21901, Wako Pure Chemical Industries). A 1:10,000 dilution of the horseradish peroxidase-linked anti-mouse IgG antibody (NA931, GE Healthcare) was used as a secondary antibody. Protein blots were visualized using the horseradish peroxidase-based ECL Plus reagent (GE Healthcare) with an Image Quant LAS 4010 biomolecular imager (GE Healthcare).

Yeast two-hybrid analysis. The GAL4-based yeast two-hybrid system (Matchmaker Gold Yeast Two-Hybrid System, Clontech) was used for detecting proteinprotein interactions. Full-length, N-terminal half (amino acids 1-392) and C-terminal half (amino acids 383-1,187) MpGI cDNAs were PCR-amplified and subcloned into the EcoRI-SalI site of pGBKT7 (Clontech). Truncated cDNA clones of MPFKF containing full length, LOV domain + F-box domain (amino acids 1291 ) and F-box domain + Kelch repeats (181-635) were PCR amplified and subcloned into the NdeI-BamHI site of pGADT7 (Clontech). After the sequences were confirmed, the plasmids were transformed into the yeast strain AH109 and transformants selected on SD - WL medium. The resulting colonies were cultured in SD - WL liquid medium and then serially diluted in distilled water before being spotted onto $S D-W L$ and $S D-W L H$ plates to analyse the interaction. pGBKT7 and pGADT7 plasmids were used as negative controls for yeast two-hybrid analysis. After a 2- to 3 -day-incubation at $28^{\circ} \mathrm{C}$, the plates were photographed. The experiments were repeated three times with similar results. The gene-specific primer sets used for the generation of each construct are given in Supplementary Table 1.

Transformation of $\mathbf{M p G I}$ into an A. thaliana gi mutant. The upstream regions of AtGI including the promoter and $5^{\prime} \mathrm{UTR}(2,106 \mathrm{bp})$ and the full-length cDNA of $M p G I$ were amplified by PCR with the primer pairs: forward 5'-CACCTGCCTA CAATTTGAGAATACGCC- $3^{\prime}$, reverse $5^{\prime}$-TTCTGCCCGGGAAATGACATCCAG GAACCGAAACTAAACCCCAAC- $3^{\prime}$ for the upstream regions of AtGI, and forward 5'-GGTTTAGTTTCGGTTCCTGGATGTCATTTCCCGGGCAGAAAT GGC- $3^{\prime}$ and reverse $5^{\prime}$-TTAGACAGGAATCGGGCAGC- $3^{\prime}$ for $M p G I$ cDNA. The PCR products were then subjected to PCR using the primer pairs: forward $5^{\prime}$-CAC CTGCCTACAATTTGAGAATACGCC- $3^{\prime}$ and reverse $5^{\prime}$-TTAGACAGGAATCG GGCAGC- $3^{\prime}$ to generate the AtGIpro:MpGI fused sequence. The resultant fragment was introduced into pGWB1 (ref. 39) to generate the AtGIpro:MpGI 

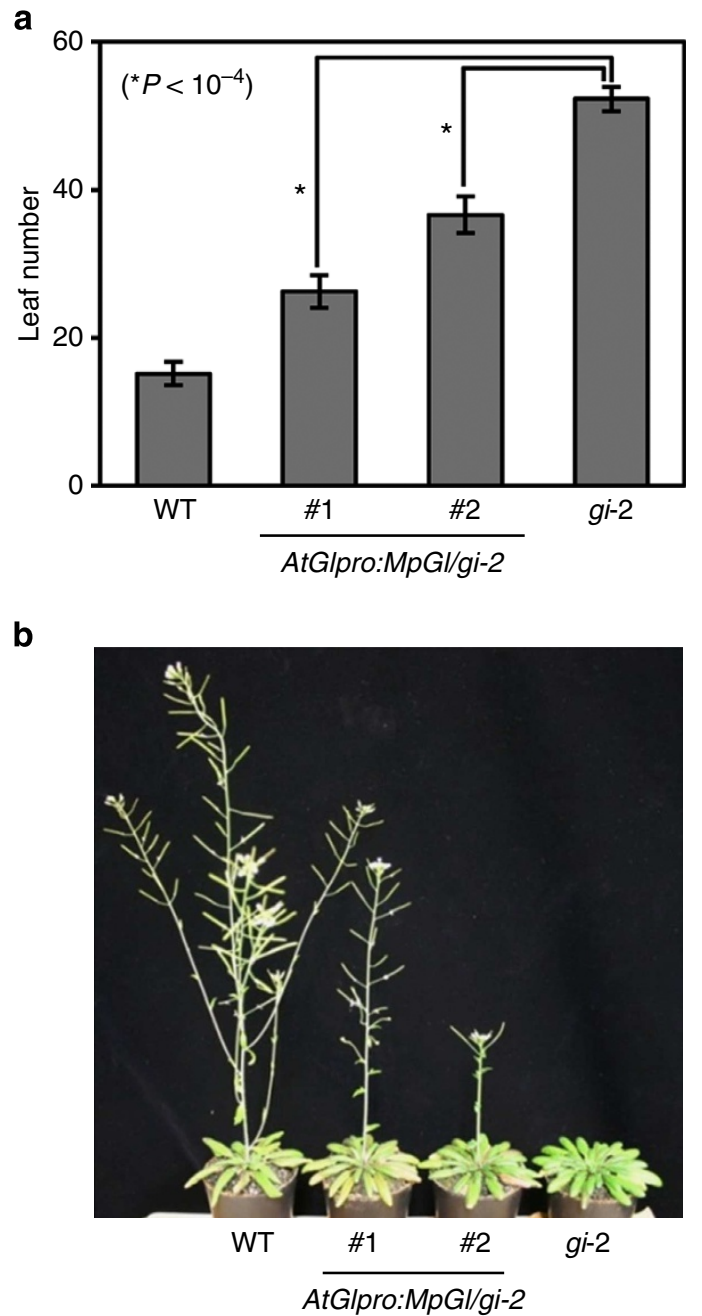

Figure 6 | Genetic complementation of the A. thaliana gi mutant by MpGI. (a) Rosette leaf numbers of wild-type A. thaliana (Columbia-0), gi-2, and gi-2 harbouring an AtGlpro:MpGl construct, at the time of bolting under longday conditions. ${ }^{*} P<10^{-4}$. Error bars, s.d.; $n>10$. $P$-values were determined using unpaired Student's t-test. (b) Representative individuals of wild type, gi-2, and AtGlpro:MpGI/gi-2 at 45 days after germination. WT, wild type.

binary plasmid. pGWB1 were kindly provided by T. Nakagawa (Shimane University). The construct was introduced into the A. thaliana gi-2 mutant using the floral dip method ${ }^{40}$. Transgenic lines that possessed a single copy of the AtGIpro:MpGI construct in gi-2 were used for further analysis. For flowering phenotype analysis, plants were grown on soil in growth chambers (LH300, NKSystem) under 16-h-light/8-h-dark conditions with an illumination rate of 90 to $110 \mu \mathrm{mol} \mathrm{m}^{-2} \mathrm{~s}^{-1}$ of white fluorescent light (FL40SSEX-N/37-HG, NEC Lighting) at $22{ }^{\circ} \mathrm{C}$. The Columbia- 0 accession was used as the wild type. Flowering time was measured by counting the number of rosette leaves upon bolting.

Histochemical GUS staining. The MpGIpro:GUS- and MpFKFpro:GUS-expressing plants were grown on half-strength B5 agar plates containing $1 \%$ sucrose for 10 days under continuous light. Histochemical GUS staining was performed as described previously ${ }^{41}$. The staining patterns of GUS activity from more than 10 individual lines were analysed. Representative patterns are shown in Fig. 4.

\section{References}

1. Song, Y. H., Ito, S. \& Imaizumi, T. Flowering time regulation: photoperiod- and temperature-sensing in leaves. Trends Plant Sci. 18, 575-583 (2013).

2. Song, Y. H., Ito, S. \& Imaizumi, T. Similarities in the circadian clock and photoperiodism in plants. Curr. Opin. Plant Biol. 13, 594-603 (2010).

3. Itoh, H. \& Izawa, T. The coincidence of critical day length recognition for florigen gene expression and floral transition under long-day conditions in rice. Mol. Plant 6, 635-649 (2013).
4. Sawa, M., Nusinow, D. A., Kay, S. A. \& Imaizumi, T. FKF1 and GIGANTEA complex formation is required for day-length measurement in Arabidopsis. Science 318, 261-265 (2007).

5. Imaizumi, T., Tran, H. G., Swartz, T. E., Briggs, W. R. \& Kay, S. A. FKF1 is essential for photoperiodic-specific light signalling in Arabidopsis. Nature 426, 302-306 (2003).

6. Imaizumi, T., Schultz, T. F., Harmon, F. G., Ho, L. A. \& Kay, S. A. FKF1 F-box protein mediates cyclic degradation of a repressor of CONSTANS in Arabidopsis. Science 309, 293-297 (2005).

7. Fornara, F. et al. Arabidopsis DOF transcription factors act redundantly to reduce CONSTANS expression and are essential for a photoperiodic flowering response. Dev. Cell 17, 75-86 (2009).

8. Holm, K., Kallman, T., Gyllenstrand, N., Hedman, H. \& Lagercrantz, U. Does the core circadian clock in the moss Physcomitrella patens (Bryophyta) comprise a single loop? BMC Plant Biol. 10, 109 (2010).

9. Hayama, R., Izawa, T. \& Shimamoto, K. Isolation of rice genes possibly involved in the photoperiodic control of flowering by a fluorescent differential display method. Plant Cell Physiol. 43, 494-504 (2002).

10. Hecht, V. et al. Pea LATE BLOOMER1 is a GIGANTEA ortholog with roles in photoperiodic flowering, deetiolation, and transcriptional regulation of circadian clock gene homologs. Plant Physiol. 144, 648-661 (2007).

11. Higuchi, Y. et al. Constitutive expression of the GIGANTEA ortholog affects circadian rhythms and suppresses one-shot induction of flowering in Pharbitis nil, a typical short-day plant. Plant Cell Physiol. 52, 638-650 (2011).

12. Watanabe, S. et al. A map-based cloning strategy employing a residual heterozygous line reveals that the GIGANTEA gene is involved in soybean maturity and flowering. Genetics 188, 395-407 (2011).

13. Zhao, X. Y., Liu, M. S., Li, J. R., Guan, C. M. \& Zhang, X. S. The wheat TaGI1, involved in photoperiodic flowering, encodes an Arabidopsis GI ortholog. Plant Mol. Biol. 58, 53-64 (2005).

14. Benson-Evans, K. Physiology of the reproduction of bryophytes. Bryologist 67, 431-445 (1964).

15. Hohe, A., Rensing, S. A., Mildner, M., Lang, D. \& Reski, R. Day length and temperature strongly influence sexual reproduction and expression of a novel MADS-Box gene in the moss Physcomitrella patens. Plant Biol. 4, 762-762 (2002).

16. Benson-Evans, K. Environmental factors and bryophytes. Nature 191, 255-260 (1961).

17. Bowman, J. L., Floyd, S. K. \& Sakakibara, K. Green genes-comparative genomics of the green branch of life. Cell 129, 229-234 (2007).

18. Qiu, Y. L. et al. The deepest divergences in land plants inferred from phylogenomic evidence. Proc. Natl Acad. Sci. USA 103, 15511-15516 (2006).

19. Chiyoda, S., Ishizaki, K., Kataoka, H., Yamato, K. T. \& Kohchi, T. Direct transformation of the liverwort Marchantia polymorpha L. by particle bombardment using immature thalli developing from spores. Plant Cell Rep. 27, 1467-1473 (2008).

20. Ishizaki, K., Chiyoda, S., Yamato, K. T. \& Kohchi, T. Agrobacterium-mediated transformation of the haploid liverwort Marchantia polymorpha L., an emerging model for plant biology. Plant Cell Physiol. 49, 1084-1091 (2008)

21. Kubota, A., Ishizaki, K., Hosaka, M. \& Kohchi, T. Efficient Agrobacteriummediated transformation of the liverwort Marchantia polymorpha using regenerating thalli. Biosci. Biotechnol. Biochem. 77, 167-172 (2013).

22. Tsuboyama, S. \& Kodama, Y. AgarTrap: A simplified Agrobacterium-mediated transformation method for sporelings of the liverwort Marchantia polymorpha L. Plant Cell Physiol. 55, 229-236 (2014).

23. Ishizaki, K., Johzuka-Hisatomi, Y., Ishida, S., Iida, S. \& Kohchi, T. Homologous recombination-mediated gene targeting in the liverwort Marchantia polymorpha L. Sci. Rep. 3, 1532 (2013).

24. Sugano, S. S. et al. CRISPR/Cas9-mediated targeted mutagenesis in the liverwort Marchantia polymorpha L. Plant Cell Physiol. 55, 475-481 (2014).

25. Voth, P. D. \& Hamner, K. C. Responses of Marchantia polymorpha to nutrient supply and photoperiod. Bot. Gaz. 102, 169-205 (1940).

26. Wann, F. B. Some of the factors involved in the sexual reproduction of Marchantia polymorpha. Am. J. Bot. 12, 307-318 (1925).

27. Nelson, D. C., Lasswell, J., Rogg, L. E., Cohen, M. A. \& Bartel, B. FKF1, a clockcontrolled gene that regulates the transition to flowering in Arabidopsis. Cell 101, 331-340 (2000).

28. Schultz, T. F., Kiyosue, T., Yanovsky, M., Wada, M. \& Kay, S. A. A role for LKP2 in the circadian clock of Arabidopsis. Plant Cell 13, 2659-2670 (2001).

29. Somers, D. E., Schultz, T. F., Milnamow, M. \& Kay, S. A. ZEITLUPE encodes a novel clock-associated PAS protein from Arabidopsis. Cell 101, 319-329 (2000).

30. Fowler, S. et al. GIGANTEA: a circadian clock-controlled gene that regulates photoperiodic flowering in Arabidopsis and encodes a protein with several possible membrane-spanning domains. EMBO J. 18, 4679-4688 (1999).

31. Park, D. H. et al. Control of circadian rhythms and photoperiodic flowering by the Arabidopsis GIGANTEA gene. Science 285, 1579-1582 (1999). 
32. Althoff, F. et al. Comparison of the MpEF1 $\alpha$ and CaMV35 promoters for application in Marchantia polymorpha overexpression studies. Transgenic Res. 23, 235-244 (2014).

33. Zobell, O., Coupland, G. \& Reiss, B. The family of CONSTANS-like genes in Physcomitrella patens. Plant Biol. 7, 266-275 (2005).

34. Kardailsky, I. et al. Activation tagging of the floral inducer FT. Science 286, 1962-1965 (1999).

35. Kobayashi, Y., Kaya, H., Goto, K., Iwabuchi, M. \& Araki, T. A pair of related genes with antagonistic roles in mediating flowering signals. Science 286, 1960-1962 (1999).

36. Hedman, H., Kallman, T. \& Lagercrantz, U. Early evolution of the MFT-like gene family in plants. Plant Mol. Biol. 70, 359-369 (2009).

37. Gamborg, O. L., Miller, R. A. \& Ojima, K. Nutrient requirements of suspension cultures of soybean root cells. Exp. Cell Res. 50, 151-158 (1968).

38. Guindon, S. \& Gascuel, O. A simple, fast, and accurate algorithm to estimate large phylogenies by maximum likelihood. Syst. Biol. 52, 696-704 (2003).

39. Nakagawa, T. et al. Development of series of gateway binary vectors, pGWBs, for realizing efficient construction of fusion genes for plant transformation. J. Biosci. Bioeng. 104, 34-41 (2007).

40. Clough, S. J. \& Bent, A. F. Floral dip: a simplified method for Agrobacteriummediated transformation of Arabidopsis thaliana. Plant J. 16, 735-743 (1998).

41. Ishizaki, K., Nonomura, M., Kato, H., Yamato, K. T. \& Kohchi, T. Visualization of auxin-mediated transcriptional activation using a common auxin-responsive reporter system in the liverwort Marchantia polymorpha. J. Plant Res. 125, 643-651 (2012).

\section{Acknowledgements}

We thank Sakiko Ishida and Yoriko Matsuda for technical assistance, Makoto Shirakawa, Shohei Yamaoka and Takashi Araki for critical discussions, Motomu Endo for providing gi-2 seeds, and Tsuyoshi Nakagawa for providing the Gateway binary plasmids. This work was supported by a Grant-in-Aid for Scientific Research on Priority Area (23012025 to T.K.); Scientific Research on Innovative Area (25120716 and 25113009 to T.K.; 25119711 and 25114510 to K.I.) from the Ministry of Education, Culture, Sports, Science and Technology of Japan; a Grant-in-Aid for Scientific Research (C) (24580140 to K.I.); and Research Fellowships of the Japan Society for the Promotion of Science for Young Scientists (24-6987 to A.K.) from the Japan Society for the Promotion of Science.

\section{Author contributions}

A.K., K.I., K.T.Y. and T.K. designed the research. A.K. and S.K. performed the experiments. A.K., S.K., K.I., R.N. and T.K. analysed the data. A.K., K.I., R.N., K.T.Y. and T.K. prepared the manuscript. T.K. supervised the work.

\section{Additional information}

Accession codes: The sequence of the $M p G I$ and $M p F K F$ genes are available in DDBJ (accession numbers AB872959 for MpGI and AB872960 for MpFKF).

Supplementary Information accompanies this paper at http://www.nature.com/ naturecommunications

Competing financial interests: The authors declare no competing financial interests.

Reprints and permission information is available online at http://npg.nature.com/ reprintsandpermissions/

How to cite this article: Kubota, A. et al. Co-option of a photoperiodic growth-phase transition system during land plant evolution. Nat. Commun. 5:3668 doi: $10.1038 /$ ncomms4668 (2014). 